\title{
Toxicity of Pioglitazone on Mitochondria Isolated from Brain and Heart: An Analysis for Probable Drug-Induced Neurotoxicity and Cardiotoxicity
}

\author{
Authors \\ Enaytollah Seydi ${ }^{1,2}$, Tina Servati ${ }^{3}$, Fatemeh Samiei ${ }^{3}$, Parvaneh Naserzadeh $^{3}$, Jalal Pourahmad ${ }^{3}$
}

\author{
Affiliations \\ 1 Department of Occupational Health and Safety \\ Engineering, Alborz University of Medical Sciences, Karaj, \\ Iran (the Islamic Republic of) \\ 2 Research Center for Health, Safety and Environment, \\ Alborz University of Medical Sciences, Karaj, Iran \\ 3 Department of Pharmacology and Toxicology, \\ Shahid Beheshti University of Medical Science, Tehran, \\ Iran (the Islamic Republic of)
}

Key words

pharmacology, toxicology, apoptosis

received $\quad 03.11 .2019$

accepted $\quad 16.12 .2019$

Bibliography

DOI https://doi.org/10.1055/a-1085-2542

Published online: 13.1 .2020

Drug Res 2020; 70: 112-118

(c) Georg Thieme Verlag KG Stuttgart · New York

ISSN 2194-9379

Correspondence

Dr. Jalal Pourahmad

Department of Pharmacology and Toxicology

Shahid Beheshti University of Medical Science

23451 Tehran

Iran (the Islamic Republic of)

Tel.: 98 (21) 2255-8786, Fax : + 98 (21) 8820-9620

j.pourahmadjaktaji@utoronto.ca

\section{ABSTRACT}

Pioglitazone (PG) is one of the thiazolidinedione (TZDs) drugs used in diabetic patients. TZDs are known as peroxisome proliferator-activated receptor gamma (PPARY) agonists. Mitochondria are considered as one of the targets of these drugs. The mechanisms of the effect of PG on mitochondria are not well understood. In this study, we investigated the effect of PG on mitochondria isolated from brain and heart. Mitochondrial parameters such as succinate dehydrogenase (SDH) activity, reactive oxygen species (ROS) generation, collapse in mitochondrial membrane potential (MMP), mitochondrial swelling and cytochrome $\mathrm{c}$ release were evaluated. The results showed that PG at concentrations of $12.5,25$ and $50 \mu \mathrm{g} / \mathrm{ml}$ increased the generation of ROS, the collapse of MMP, mitochondrial swelling and the release of cytochrome $\mathrm{c}$ in mitochondria isolated from both brain and heart tissues. The underlying mechanisms of PG induced neuro-toxicity and cardio-toxicity may be associated with changes in mitochondrial function, ROS generation (oxidative stress), and changes in the mitochondrial membrane.

\section{Introduction}

The thiazolidinedione (TZDs) are used in patients with diabetes to help improve insulin resistance and glucose homeostasis. Furthermore, these drugs are known as peroxisome proliferator-activated receptor gamma (PPARY) agonists [1-4]. These synthetic compounds are pioglitazone, rosiglitazone, troglitazone and ciglitazone $[5,6]$. There are controversies in the relationship between the use of TZDs drugs, such as pioglitazone (PG), and the cardiovascular outcomes. Some research has shown that there is a positive relationship between the use of PG and heart failure [7-9]. In recent years, PG has been used to improve some brain disorders. However, little is known about its neurotoxicity [10-12].

Many compounds have caused mitochondrial toxicity and these organelles has become one of the research targets $[13,14]$. Brain and heart tissues depend on mitochondria for their high energy consumption and maintenance of their normal function $[15,16]$. Research has shown that mitochondria are one of the targets of PPARy agonists $[3,17,18]$. Mitochondrial dysfunction has been reported by exposure to TZDs. Research shows that TZDs increases the generation of reactive oxygen species (ROS) through dis- 
ruption of respiratory chain complexes I and III in mitochondria, collapse in mitochondria membrane potential (MMP), cytochrome c release, mitochondrial swelling, and apoptosis [5, 19-23]. One of the mechanisms by which TZDs cause cytotoxicity is through the generation of ROS. It has been reported that there is a direct relevance between the level of ROS and the degree of cytotoxicity induced by these compounds [2, 3, 14]. Increased ROS generation induced by some TZDs lead to oxidation of vital components within mitochondria (such as mitochondrial DNA, mtDNA) and also induction of apoptotic signaling [21]. Incubation of isolated brain mitochondria with pioglitazone resulted in impairment of complex III in the mitochondrial respiratory chain [17].

The mitochondrial organelle is considered the source and target of reactive oxygen species (ROS). The electron transport chain in mitochondria is one of the most important sources of ROS generation [24-27]. Research has shown that mitochondrial dysfunction has been implicated in the etiology of many diseases [22]. The consequences of mitochondrial inhibition or disruption include the generation of ROS, ATP depletion, and eventually cell death (apoptosis/necrosis). Mitochondria as one of the important organelles in eukaryotic cells are involved in important physiological processes including the generation of free radicals, energy production and cell death $[2,3,14,19,22]$. Therefore, mitochondrial dysfunction can be dangerous for different cells and organs due to insufficient ATP generation and excessive level of ROS [18]. The in vitro cytotoxicity investigations can be helpful in providing mechanistic information, and this information can be useful in understanding the more detailed clinical observations [19].

The effects of PG on mitochondria have not been fully studied. Therefore, we studied the response of mitochondria isolated from the rat brain and heart to several concentrations of PG by measuring succinate dehydrogenase (SDH) activity, ROS generation, MMP collapse, mitochondria swelling and cytochrome $\mathrm{c}$ release.

\section{Materials and Methods}

\section{Animals}

Male Wistar rats ( $n=10$ ), weighing 250-300 g were housed under standard conditions (temperature $20-25^{\circ} \mathrm{C}$, humidity $50-60 \%$, $12 \mathrm{~h}$ light-dark cycle and free access to food and water). The experimental protocols were approved by the Animal Ethics Committee of Shahid Beheshti University of Medical Sciences. All efforts were made to minimize the number and the suffering of animals used.

\section{Mitochondria Isolation}

In this study, mitochondria were isolated from the fresh brain and heart using a mitochondrial isolation kit from Sigma Chemical Co. (St. Louis, MO, USA) according to the manufacture's instruction. The protein concentration of the pellet mitochondria was measured using Bradford protein assay [28]. Furthermore, mitochondrial function was assessed through determining mitochondrial SDH activity, mitochondrial reactive oxygen species (ROS) level, mitochondrial membrane potential (MMP) collapse, mitochondrial swelling and cytochrome $\mathrm{c}$ release. In this study, the mitochondrial purity and integrity were performed through the MTT test (for evaluation of mitochondrial function/ mitochondrial complex II) and cytochrome c oxidase (complex IV) assay kit, respectively.

\section{Succinate Dehydrogenase (SDH) assay}

Briefly, MTT dye was used to evaluate SDH activity. At first, mitochondria isolated from the brain and heart were exposed to PG concentrations $(12.5,25$ and $50 \mu \mathrm{g} / \mathrm{ml})$ for $30 \mathrm{~min}$. Then, MTT $(0.4 \%)$ was added to the mitochondrial suspension and incubated at $37{ }^{\circ} \mathrm{C}$ for $30 \mathrm{~min}$. In the final step, dimethyl sulfoxide (DMSO, $100 \mu \mathrm{l}$ ) were used to dissolve formazan crystals, then the absorbance $(570 \mathrm{~nm})$ was assayed using an ELISA reader (Tecan, Rainbow Thermo, Austria) [29].

\section{ROS determination assay}

2,7-dichlorofluorescein diacetate (DCFH-DA) probe at final concentration of $10 \mu \mathrm{M}$ was used to evaluate mitochondrial ROS generation. The isolated mitochondria from brain and heart were suspended in respiration assay buffer and then were exposed to PG concentrations $(12.5,25$ and $50 \mu \mathrm{g} / \mathrm{ml})$. Then, DCFH-DA was added to the mitochondrial suspension and incubated for 5, 30 and $60 \mathrm{~min}$ at $37^{\circ} \mathrm{C}$. The fluorescence intensity $(\mathrm{EX} \lambda=488 \mathrm{~nm}$ and $\mathrm{EM} \lambda=527 \mathrm{~nm})$ was assayed using a fluorescence spectrophotometer (Shimadzu RF5000U) [30].

\section{MMP determination assay}

The Rhodamine 123 (Rh 123) probe at final concentration of $10 \mu \mathrm{M}$ was used to evaluate MMP collapse. The isolated mitochondria from brain and heart were suspended in MMP assay buffer and then were exposed to PG concentrations (12.5, 25 and $50 \mu \mathrm{g} / \mathrm{ml})$. Then, Rh 123 was added to the mitochondrial suspension and incubated for 5,30 and $60 \mathrm{~min}$ at $37^{\circ} \mathrm{C}$. The fluorescence intensity $(\mathrm{EX} \lambda=490 \mathrm{~nm}$ and $E M \lambda=530 \mathrm{~nm}$ ) was assayed using a fluorescence spectrophotometer (Shimadzu RF5000U) [31].

\section{Mitochondrial swelling}

The isolated mitochondria from brain and heart were suspended in mitochondrial swelling assay buffer and then were exposed to PG concentrations $(12.5,25$ and $50 \mu \mathrm{g} / \mathrm{ml})$. Then, mitochondrial swelling was evaluated at 5,30 and $60 \mathrm{~min}$ at $37{ }^{\circ} \mathrm{C}$. The absorbance $(540 \mathrm{~nm})$ was assayed using using an ELISA reader (Tecan, Rainbow Thermo, Austria) [30].

\section{Cytochrome $\mathrm{c}$ release}

Briefly, cytochrome c release was evaluated using the Quantikine Rat/Mouse Cytochrome c Immunoassay kit provided by R \& D Systems, Inc. (Minneapolis, Minn.). The micro-plate was used to precoating the monoclonal antibody specific for rat/mouse cytochrome c. In the next step, conjugate $(75 \mu \mathrm{L})$, standard and positive control $(50 \mu \mathrm{L})$ were added to each well of the micro-plate. Then, $1 \mu \mathrm{g}$ of protein from each supernatant fraction was added to the sample wells. All controls and standards, controls and samples were added to the micro-plate (two wells), and then substrate solution $(100 \mu \mathrm{l})$ was added to micro-plate. Finally, stop solution $(100 \mu \mathrm{l})$ was added to each well of micro-plate and optical density was evaluated at $540 \mathrm{~nm}$. 


\section{Statistical analysis}

Results are presented as mean \pm SD. All statistical analyses were performed using GraphPad Prism (version 5). The assays were performed 3 times. Statistical significance was determined using the one-way ANOVA test, followed by the post hoc Tukey test. The oneway ANOVA test was used as a specific statistical analysis for the determinations of SDH activity, and cytochrome c release. In some experiments, the two-way ANOVA test, followed by the post hoc Bonferroni test was also performed. The two-way ANOVA test was used for the determinations of mitochondrial ROS level, MMP and mitochondrial swelling. Statistical significance was set at $P<0.05$.

\section{Results}

\section{PG decreased the SDH activity}

The results showed that exposure to PG $(12.5,25$ and $50 \mu \mathrm{g} / \mathrm{ml})$ decreased SDH activity in mitochondria isolated from the brain and heart ( $>$ Fig. 1). Also, this decrease in SDH activity in mitochondria isolated was in a concentration-dependent pattern $(50>25>12.5 \mu \mathrm{g} / \mathrm{ml})$. In fact, the decrease in absorbance indicates a decrease in SDH activity.

\section{PG increased the ROS generation}

In $>$ Fig. 2, exposure of mitochondria isolated from the brain ( $\triangleright$ Fig. 2a) and heart ( $\vee$ Fig. 2 b) to PG at all applied concentrations $(12.5,25$ and $50 \mu \mathrm{g} / \mathrm{ml})$ showed an increase in the level of ROS generation. PG in a dose- and concentration-dependent manner increased the level of ROS generation in isolated the brain and heart mitochondria.

\section{PG increased the MMP collapse}

The results in $>$ Fig. 3 a show that PG was able to collapse on MMP at concentrations of 25 and $50 \mu \mathrm{g} / \mathrm{ml}$ in the isolated mitochondria from the brain and at concentration of $12.5 \mu \mathrm{g} / \mathrm{ml}$ had no effect on
MMP. However, PG at all concentrations caused the collapse of the MMP in mitochondria isolated from the heart.

\section{PG increased the mitochondrial swelling}

The results showed that exposure to PG $(12.5,25$ and $50 \mu \mathrm{g} / \mathrm{ml})$ at 5,30 and 60 min increased mitochondrial swelling in mitochondria isolated from the brain ( $\vee$ Fig. $\mathbf{4 a}$ ) and heart ( $\triangleright$ Fig. 4 b). Also, this

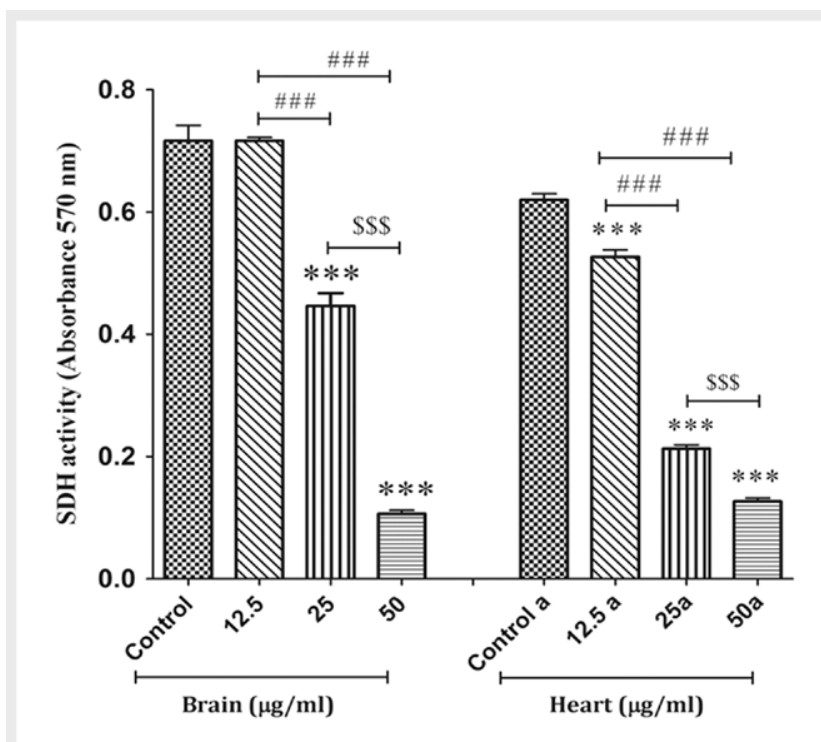

- Fig. 1 SDH activity assay. The effect of PG on the SDH activity in the mitochondria isolated from the brain and heart. Data are presented as mean $\pm S D(n=3)$. The one-way ANOVA test was carried out. ${ }^{* * *}$ show a significant difference in comparison with the corresponding control group $(P<0.001)$. \#\#\# Show a significant difference between $12.5 \mu \mathrm{g} / \mathrm{ml}$ group 25 and $50 \mu \mathrm{g} / \mathrm{ml}(P<0.001)$ groups. $\$ \$ \$$ Show a significant difference between $25 \mu \mathrm{g} / \mathrm{ml}$ group $50 \mu \mathrm{g} / \mathrm{ml}$ group $(P<0.001)$.

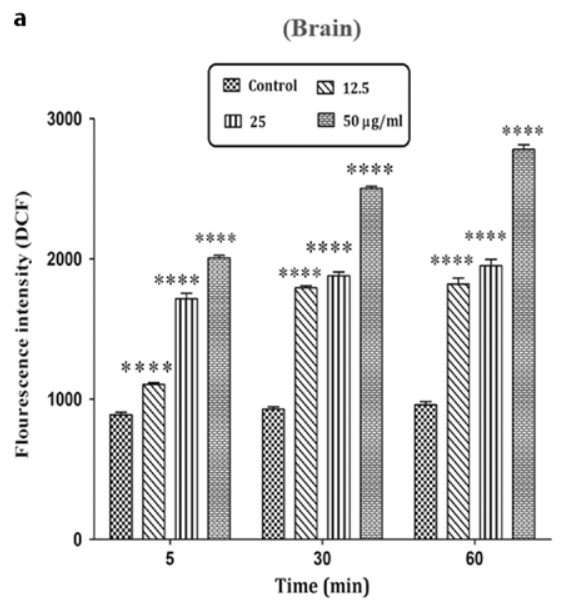

b

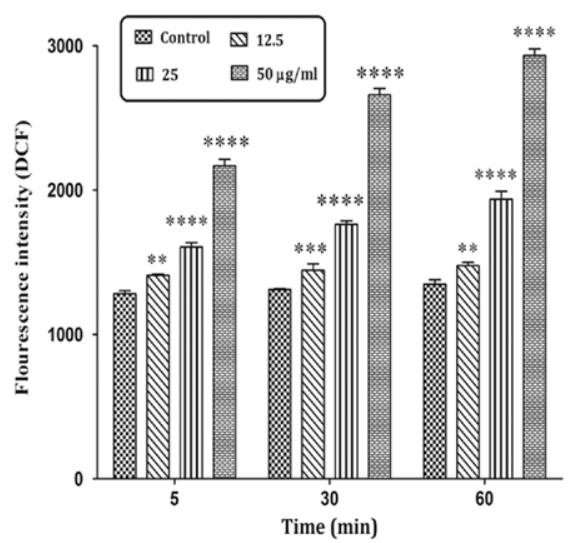

Fig. 2 ROS generation assay. The effect of PG on the ROS generation in the mitochondria isolated from the brain a and heart b. Data are presented as mean \pm SD $(n=3)$. The two-way ANOVA test was carried out. " * and ${ }^{* * * *}$ show a significant difference in comparison with the corresponding control group $(\mathrm{P}<0.01$ and $\mathrm{P}<0.0001$, respectively). 

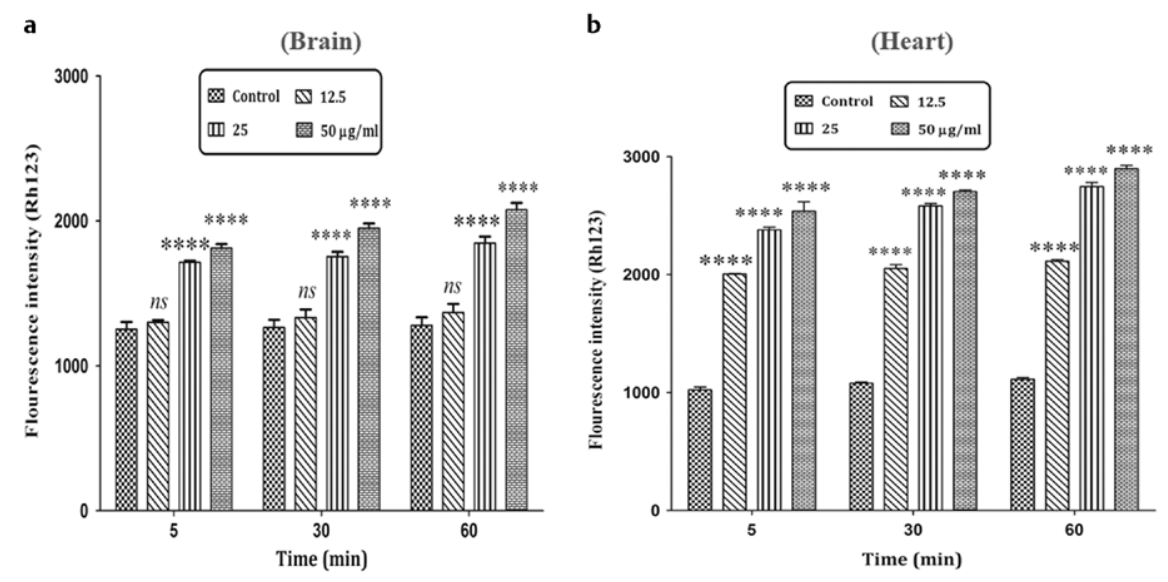

-Fig. 3 MMP collapse assay. The effect of PG on the MMP collapse in the mitochondria isolated from the brain $\mathbf{a}$ and heart $\mathbf{b}$. Data are presented as mean $\pm S D(n=3)$. The two-way ANOVA test was carried out. ${ }^{* * * *}$ show a significant difference in comparison with the corresponding control group $(P<0.0001)$.
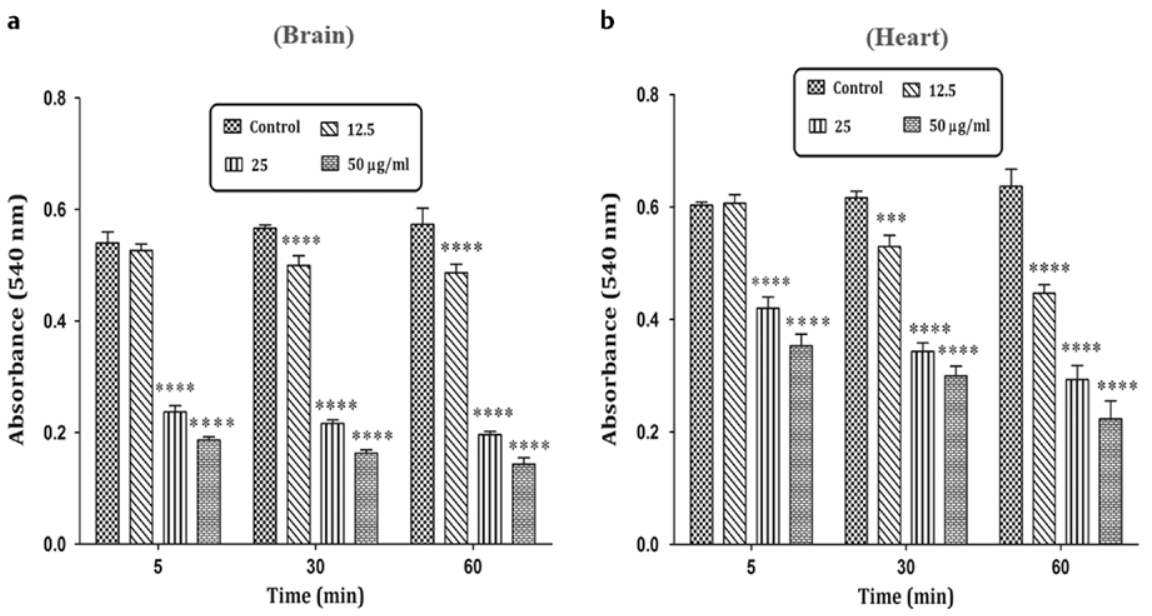

-Fig. 4 Mitochondrial swelling assay. The effect of PG on the mitochondrial swelling in the mitochondria isolated from the brain $\mathbf{a}$ and heart $\mathbf{b}$. Data are presented as mean $\pm S D(n=3)$. The two-way ANOVA test was carried out. * * * show a significant difference in comparison with the corresponding control group $(\mathrm{P}<0.0001)$.

increase in mitochondrial swelling in mitochondria isolated was in a concentration-dependent pattern $(50>25>12.5 \mu \mathrm{g} / \mathrm{ml})$. In fact, the decrease in absorbance indicates an increase in mitochondrial swelling.

\section{PG increased the cytochrome c release}

In $>$ Fig. 5, exposure of mitochondria isolated from the brain ( $>$ Fig. 5a) and heart ( $\vee$ Fig. 5b) to PG at all applied concentrations $(12.5,25$ and $50 \mu \mathrm{g} / \mathrm{ml})$ showed an increase in the release of cytochrome c.

Considerably, the pretreatment of mitochondria with the MPT inhibitor (cyclosporine A; Cs A) and an antioxidant (butylated hydroxytoluene; BHT), inhibited cytochromec release from PG $(25 \mu \mathrm{g} / \mathrm{ml})$ treated mitochondria. Our results showed that PG release of cytochrome $\mathrm{c}$ due to oxidative stress and MPT pore opening.

\section{Discussion}

Today, PG is used in the treatment of hyperglycemia in diabetic patients (type-2) [10,32]. Research has shown that some TZDs induce mitochondrial dysfunction through different mechanisms, including increase in ROS level, collapse in the MMP, mitochondrial swelling, and induction of apoptosis signaling [5, 19-23]. However, mechanistic information regarding exposure to PG and mitochondrial dysfunction is not available. In this study, we investigated mitochondrial function after exposure to different concentrations of 
a

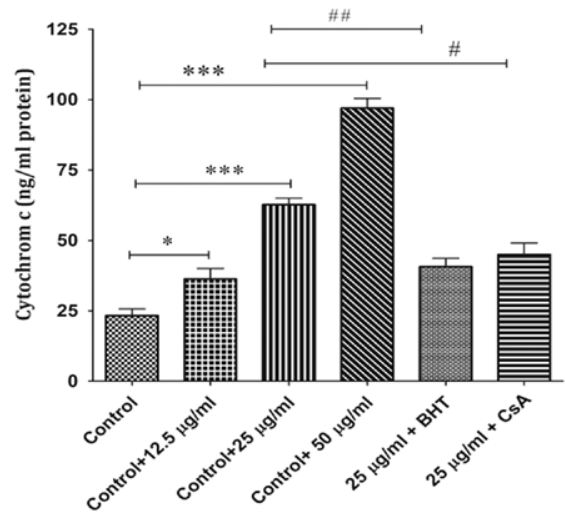

b

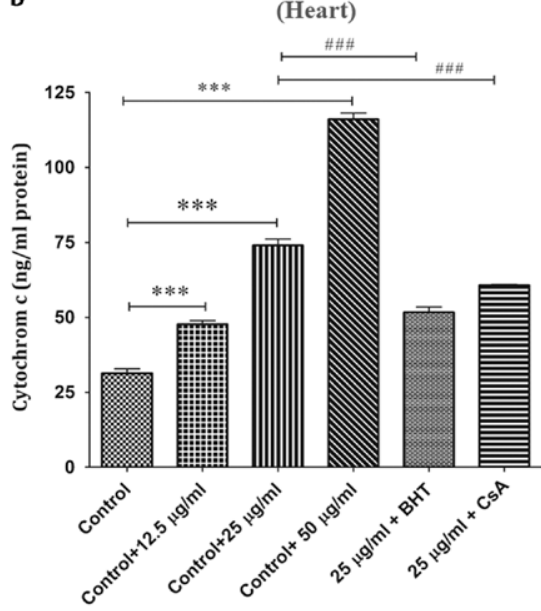

- Fig. 5 Cytochrome $\mathrm{c}$ release assay. The effect of PG on the cytochrome $\mathrm{c}$ release assay in the mitochondria isolated from the brain $\mathbf{a}$ and heart $\mathbf{b}$. Data are presented as mean $\pm S D(n=3)$. The one-way ANOVA test was carried out. " and * * " show a significant difference in comparison with the corresponding control group $(\mathrm{P}<0.05$ and $\mathrm{P}<0.001)$. \#, \#\# and \#\# show a significant difference between the control plus $25 \mu \mathrm{g} / \mathrm{ml}$ with $25 \mu \mathrm{g} / \mathrm{ml} \mathrm{plus}$ BHT and $25 \mu \mathrm{g} / \mathrm{ml}$ plus CsA $(\mathrm{P}<0.05, \mathrm{P}<0.01$ and $\mathrm{P}<0.001)$.

PG. Furthermore, the functions of freshly mitochondria (mitochondria isolated from the rat brain and heart) were assessed by measuring SDH activity, ROS generation, MMP collapse, mitochondrial swelling, and cytochrome $\mathrm{c}$ release.

The brain tissue holds nearly $2 \%$ of total body mass, but consumes nearly $20 \%$ of total body energy (ATP). In fact, the brain is one of the tissues that needs a lot of ATP. Mitochondrion is known as a source of energy in the body, and it produces energy through the respiratory chain. Therefore, brain tissue needs mitochondria to maintain its normal function and energy consumption. Mitochondrial dysfunction in the brain is associated with neurodegenerative diseases [15, 33, 34]. In addition, energy consumption in the heart is similar to that of the brain. On the other hand, the heart needs energy for its normal function and development, and mitochondria are the source of this energy $[16,35,36]$.

In the mitochondria isolated from the brain and heart, we found a significant decrease in SDH activity compared with control group following the addition of several concentrations of PG. ROS are involved in important physiological processes including cell growth and proliferation and apoptosis. Studies have shown that mitochondria are a major source of ROS. The generation of ROS is via the electron leakage in the respiratory chain of mitochondria, especially complexes I and III [24, 37-39]. High levels of oxygen consumption can lead to increase generation of ROS in tissues with high oxygen consumption (such as brain and heart). High levels of free radicals lead to consequences including oxidative stress, damage to the mitochondrial membrane and mtDNA, and induction of apoptosis [40-42]. Our findings regarding ROS generation using DCFH-DA shows that PG increases the ROS levels in the mitochondria obtained from the brain and heart at 5, 30 and 60 min after exposure. The results of this study are in agreement with other studies that have shown that exposure to some TZDs increases the generation of ROS $[3,14]$.
An increase in the level of ROS can induce the opening of mitochondrial permeability transition (MPT) pore [2]. Research has shown that the opening of the MPT pore in the inner membrane leads to the collapse of mitochondrial membrane potential, mitochondrial swelling, cytochrome $c$ release and subsequently induction of cell death (apoptosis) [4, 13]. The MMP as one of the most important indicators of mitochondrial function can be evaluated by fluorescence probes. Compared with the control group, exposure the mitochondria obtained from the brain and heart with PG induced significant collapse in MMP. These results are in agreement with the results of previous studies $[2,14,20,21]$. The collapse in MMP facilitates cytochrome c exit from mitochondria and induces cell death [43].

Finally, the results showed that exposure of mitochondria isolated from the brain and heart to PG caused mitochondrial swelling and cytochrome $c$ release. Cytochrome $c$ release from mitochondria is one of the early events in the cell death process [43]. In conclusion, the results of this study suggest that pioglitazone increases the generation of ROS through the effect on the mitochondrial respiratory chain. An increase in the level of ROS can induce the opening of MPT pore. Finally, the opening of MPT pore can disrupt the mitochondrial membrane, mitochondrial swelling, and cytochrome $\mathrm{c}$ release and eventually cell death in the mitochondria isolated from the brain and heart. Mitochondrial dysfunction in brain and heart is associated with neurodegenerative and cardiovascular diseases.

\section{Acknowledgements}

The results presented in this article were partly extracted from the thesis of Dr. Tina Servati (Pharm.D. graduate of Faculty of Pharmacy, Shahid Beheshti University of Medical Sciences) who performed her thesis under supervision of Prof. Jalal Pourahmad. The investi- 
gation was carried out in Prof. J. Pourahmad's laboratory in the Faculty of Pharmacy, Shahid Beheshti university of Medical Sciences, Tehran, Iran.

\section{Conflict of Interest}

The authors report no conflicts of interest.

\section{References}

[1] Divakaruni AS, Wiley SE, Rogers GW et al. Thiazolidinediones are acute, specific inhibitors of the mitochondrial pyruvate carrier. Proc Natl Acad Sci USA 2013; 110: 5422-5427

[2] Hu D, Wu CQ, Li Z] et al. Characterizing the mechanism of thiazolidinedione-induced hepatotoxicity: An in vitro model in mitochondria. Toxicol Appl Pharmacol 2015; 284: 134-141

[3] Perez-Ortiz JM, Tranque P, Vaquero CF et al. Glitazones differentially regulate primary astrocyte and glioma cell survival. Involvement of reactive oxygen species and peroxisome proliferator-activated receptor-gamma. J Biol Chem 2004; 279: 8976-8985

[4] Sanz MN, Sanchez-Martin C, Detaille D et al. Acute mitochondrial actions of glitazones on the liver: A crucial parameter for their antidiabetic properties. Cell Physiol Biochem 2011; 28: 899-910

[5] $\mathrm{He} \mathrm{H}$, Tao $\mathrm{H}$, Xiong $\mathrm{H}$ et al. Rosiglitazone causes cardiotoxicity via peroxisome proliferator-activated receptor gamma-independent mitochondrial oxidative stress in mouse hearts. Toxicol Sci 2014; 138: 468-481

[6] Skov V, Glintborg D, Knudsen S et al. Pioglitazone enhances mitochondrial biogenesis and ribosomal protein biosynthesis in skeletal muscle in polycystic ovary syndrome. PLoS One 2008; 3: e2466

[7] Giles TD, Miller AB, Elkayam U et al. Pioglitazone and heart failure: Results from a controlled study in patients with type 2 diabetes mellitus and systolic dysfunction. Journal of Cardiac Failure 2008; 14: 445-452

[8] Liao H-W, Saver JL, Wu Y-L et al. Pioglitazone and cardiovascular outcomes in patients with insulin resistance, pre-diabetes and type 2 diabetes: a systematic review and meta-analysis. BMJ Open 2017; 7: e013927

[9] Nesto RW, Bell D, Bonow RO et al. Thiazolidinedione use, fluid retention, and congestive heart failure: A consensus statement from the American Heart Association and American Diabetes Association. Circulation 2003; 108: 2941-2948

[10] Beheshti F, Hosseini M, Hashemzehi M et al. The effects of PPAR-gamma agonist pioglitazone on hippocampal cytokines, brain-derived neurotrophic factor, memory impairment, and oxidative stress status in lipopolysaccharide-treated rats. Iran J Basic Med Sci 2019; 22: 940-948

[11] McGuire JL, Correll EA, Lowery AC et al. Pioglitazone improves working memory performance when administered in chronic TBI. Neurobiol Dis 2019; 132: 104611

[12] Quan Q, Qian Y, Li X et al. Pioglitazone Reduces beta Amyloid Levels via Inhibition of PPARgamma Phosphorylation in a Neuronal Model of Alzheimer's Disease. Front Aging Neurosci 2019; 11: 178

[13] Pourahmad J, Hosseini MJ. Application of isolated mitochondria in toxicological and clinical studies. Iran J Pharm Res 2012; 11: 703-704

[14] Bova MP, Tam D, McMahon G et al. Troglitazone induces a rapid drop of mitochondrial membrane potential in liver HepG2 cells. Toxicol Lett 2005; 155: 41-50
[15] Castro JP, Wardelmann K, Grune T et al. Mitochondrial Chaperones in the Brain: Safeguarding Brain Health and Metabolism? Front Endocrinol (Lausanne) 2018; 9: 196

[16] Zhao Q, Sun Q, Zhou L et al. Complex Regulation of Mitochondrial Function During Cardiac Development. J Am Heart Assoc 2019; 8: e012731

[17] Dello Russo C, Gavrilyuk V, Weinberg G et al. Peroxisome proliferatoractivated receptor gamma thiazolidinedione agonists increase glucose metabolism in astrocytes. J Biol Chem 2003; 278: 5828-5836

[18] Sun L, Yuan Q, Xu T et al. Pioglitazone improves mitochondrial function in the remnant kidney and protects against renal fibrosis in 5/6 nephrectomized rats. Front Pharmacol 2017; 8: 545

[19] Masubuchi Y, Kano S, Horie T. Mitochondrial permeability transition as a potential determinant of hepatotoxicity of antidiabetic thiazolidinediones. Toxicology 2006; 222: 233-239

[20] Quintanilla RA, Jin YN, Fuenzalida K et al. Rosiglitazone treatment prevents mitochondrial dysfunction in mutant huntingtin-expressing cells: Possible role of peroxisome proliferator-activated receptor-gamma (PPARgamma) in the pathogenesis of Huntington disease. | Biol Chem 2008; 283: 25628-25637

[21] Rachek LI, Yuzefovych LV, Ledoux SP et al. Troglitazone, but not rosiglitazone, damages mitochondrial DNA and induces mitochondrial dysfunction and cell death in human hepatocytes. Toxicol Appl Pharmacol 2009; 240: 348-354

[22] Rezaiean Mehrabadi A, Jamshidzadeh A, Rashedinia M et al. Study of the effects of ATP suppliers and thiol reductants on toxicity of pioglitazone in isolated rat liver mitochondria. Iran J Pharm Res 2015; 14: $825-832$

[23] Shannon CE, Daniele G, Galindo C et al. Pioglitazone inhibits mitochondrial pyruvate metabolism and glucose production in hepatocytes. Febs J 2017; 284: 451-465

[24] Munro D, Treberg JR. A radical shift in perspective: Mitochondria as regulators of reactive oxygen species. J Exp Biol 2017; 220: 1170-1180

[25] Murphy MP. How mitochondria produce reactive oxygen species. Biochem J 2009; 417: 1-13

[26] Rigoulet M, Yoboue ED, Devin A. Mitochondrial ROS generation and its regulation: Mechanisms involved in $\mathrm{H}(2) \mathrm{O}(2)$ signaling. Antioxid Redox Signal 2011; 14: 459-468

[27] Zhao RZ, Jiang S, Zhang L et al. Mitochondrial electron transport chain, ROS generation and uncoupling (Review). Int J Mol Med 2019; 44: 3-15

[28] Bradford MM. A rapid and sensitive method for the quantitation of microgram quantities of protein utilizing the principle of protein-dye binding. Analytical Biochemistry 1976; 72: 248-254

[29] Zhao Y, Ye L, Liu H et al. Vanadium compounds induced mitochondria permeability transition pore (PTP) opening related to oxidative stress. J Inorg Biochem 2010; 104: 371-378

[30] Salimi A, Eybagi S, Seydi E et al. Toxicity of macrolide antibiotics on isolated heart mitochondria: A justification for their cardiotoxic adverse effect. Xenobiotica 2016; 46: 82-93

[31] Baracca A, Sgarbi G, Solaini G et al. Rhodamine 123 as a probe of mitochondrial membrane potential: Evaluation of proton flux through F(0) during ATP synthesis. Biochim Biophys Acta 2003; 1606: 137-146

[32] Hsiao PJ, Chiou HC, jiang HJ et al. Pioglitazone enhances cytosolic lipolysis, beta-oxidation and autophagy to ameliorate hepatic steatosis. Sci Rep 2017; 7: 9030

[33] Picard M, McEwen BS. Mitochondria impact brain function and cognition. Proc Natl Acad Sci USA 2014; 111: 7-8

[34] Rango M, Bresolin N. Brain Mitochondria, Aging, and Parkinson's Disease. Genes (Basel) 2018; 9: 5

[35] Griffiths E]. Mitochondria and heart disease. Adv Exp Med Biol 2012; 942: $249-267$ 
[36] Siasos G, Tsigkou V, Kosmopoulos M et al. Mitochondria and cardiovascular diseases-from pathophysiology to treatment. Ann Transl Med 2018; 6: 256

[37] Dan Dunn J, Alvarez LA, Zhang X et al. Reactive oxygen species and mitochondria: A nexus of cellular homeostasis. Redox Biol 2015; 6: 472-485

[38] Sena LA, Chandel NS. Physiological roles of mitochondrial reactive oxygen species. Mol Cell 2012; 48: 158-167

[39] Zorov DB, Juhaszova M, Sollott S]. Mitochondrial reactive oxygen species (ROS) and ROS-induced ROS release. Physiol Rev 2014; 94: 909-950
[40] Nissanka N, Moraes CT. Mitochondrial DNA damage and reactive oxygen species in neurodegenerative disease. FEBS Lett 2018; 592: 728-742

[41] Seydi E, Rasekh HR, Salimi A et al. Selective toxicity of apigenin on cancerous hepatocytes by directly targeting their mitochondria. Anticancer Agents Med Chem 2016; 16: 1576-1586

[42] Stefanatos R, Sanz A. The role of mitochondrial ROS in the aging brain. FEBS Lett 2018; 592: 743-758

[43] Gottlieb E, Armour SM, Harris MH et al. Mitochondrial membrane potential regulates matrix configuration and cytochrome $c$ release during apoptosis. Cell Death Differ 2003; 10: 709-717 